\title{
Label-Free, Real-Time Measurement of Metabolism of Adherent and Suspended Single Cells by In-Cell Fourier Transform Infrared Microspectroscopy
}

\author{
Tommaso Vannocci ${ }^{1}$, Luca Quaroni ${ }^{2, *}$ (D) Antonio de Riso ${ }^{3}$, Giulia Milordini ${ }^{1}$, Magda Wolna ${ }^{4}$, \\ Gianfelice Cinque ${ }^{4}$ (i) and Annalisa Pastore ${ }^{1, *(1)}$ \\ 1 UK Dementia Research Institute at The Wohl Institute of King's College London, London SE5 9RT, UK; \\ tommaso.vannocci@kcl.ac.uk (T.V.); giulia.milordini@iit.it (G.M.) \\ 2 Department of Physical Chemistry and Electrochemistry, Faculty of Chemistry, Jagiellonian University, \\ 30-386 Kraków, Poland \\ 3 Evotec (UK) Ltd., Dorothy Crowfoot Hodgkin Campus, Milton Park, Abingdon OX14 4RZ, UK; \\ antonio.de.riso.bioch@gmail.com \\ 4 MIRIAM beamline B22, Diamond Light Source, Harwell Campus, Didcot OX11 0DE, UK; \\ magda.wolna@yahoo.co.uk (M.W.); gianfelice.cinque@diamond.ac.uk (G.C.) \\ * Correspondence: luca.quaroni@uj.edu.pl (L.Q.); annalisa.pastore@crick.ac.uk (A.P.)
}

Citation: Vannocci, T.; Quaroni, L.; de Riso, A.; Milordini, G.; Wolna, M.; Cinque, G.; Pastore, A. Label-Free, Real-Time Measurement of

Metabolism of Adherent and Suspended Single Cells by In-Cell Fourier Transform Infrared Microspectroscopy. Int. J. Mol. Sci. 2021, 22, 10742. https://doi.org/ $10.3390 /$ ijms221910742

Academic Editors: Ian A. Nicholls and Vladimir N. Uversky

Received: 22 August 2021

Accepted: 23 September 2021

Published: 4 October 2021

Publisher's Note: MDPI stays neutral with regard to jurisdictional claims in published maps and institutional affiliations.

Copyright: (c) 2021 by the authors. Licensee MDPI, Basel, Switzerland. This article is an open access article distributed under the terms and conditions of the Creative Commons Attribution (CC BY) license (https:// creativecommons.org/licenses/by/ $4.0 /)$.
Abstract: We used infrared (IR) microscopy to monitor in real-time the metabolic turnover of individual mammalian cells in morphologically different states. By relying on the intrinsic absorption of mid-IR light by molecular components, we could discriminate the metabolism of adherent cells as compared to suspended cells. We identified major biochemical differences between the two cellular states, whereby only adherent cells appeared to rely heavily on glycolytic turnover and lactic fermentation. We also report spectroscopic variations that appear as spectral oscillations in the IR domain, observed only when using synchrotron infrared radiation. We propose that this effect could be used as a reporter of the cellular conditions. Our results are instrumental in establishing IR microscopy as a label-free method for real-time metabolic studies of individual cells in different morphological states, and in more complex cellular ensembles.

Keywords: cellular metabolism; glycolysis; infrared microscopy; synchrotron infrared; cellular adhesion

\section{Introduction}

One facet of cellular metabolism that is receiving increasing attention is the relationship between cellular morphology, adhesion, and metabolic state of the cell. It is nowadays recognized that metabolic changes accompany the morphological evolution of a cell and its interaction with the extracellular microenvironment [1,2]. Recent advances have related the metastatic capacity of cancer cells to changes in their balance between glycolysis and oxidative phosphorylation that correlate with adhesion [3,4]. Addressing the interplay of cellular metabolism and morphology calls for analytical methods with the capability to quantitatively monitor metabolite concentration in time and space across living cells at single-cell resolution. Most conventional methods for metabolic investigations require the use of bulk samples, which implies, by definition, sample averaging and prevents the possibility to associate metabolic activity to a specific cellular entity. Techniques that allow the imaging of cellular metabolism often require some form of labelling or sample enrichment, which restrict the investigation to pre-selected targets. Mass spectrometry provides the highest sensitivity and specificity, but the application to live cell sampling, using ambient ionization methods, requires at last partial cytoplasmic extraction, preventing recording of time-resolved events [5]. An optimal method should be quantitative, non-invasive, require minimal or no sample pre-treatment, and be applicable to cell culture models and living organisms. It should afford molecular specificity and the capability to 
simultaneously detect multiple molecular species, ideally without a priori target selection. Finally, it should also provide the possibility to monitor the activity of both single cells and cellular ensembles in real time. Most of the desired features are provided by mid-infrared (IR) absorption spectromicroscopy [6].

Absorption spectroscopy in the mid-IR spectral region (wavelength approx. 2.5-25 $\mu \mathrm{m}$; from now on, simply IR spectroscopy) measures vibrational transitions in matter, providing information on composition, structure, and dynamics at the molecular level. Because of its compositional sensitivity, it is often used for analytical applications in the biological sciences. In addition, its sensitivity to molecular structure and its applicability to a range of sampling conditions have been extensively utilized for the study of biomolecules and their dynamics in bulk samples.

The combination of IR spectroscopy with microscopy has enhanced the method by providing spatial information $[7,8]$, bringing the added value of microscale resolution in the spatial distribution of molecular composition and structure. The possibility of using synchrotron radiation sources coupled to Fourier Transform IR (FTIR) has further widened applications by increasing the spectral quality (signal-to-noise ratio) at the highest spatial resolution (diffraction-limited) optically allowed in IR microanalysis [9].

Spectromicroscopy in the mid-IR spectral region (from now on, simply called IR microscopy) was adapted to gain both qualitative and quantitative information on complex biological systems, such as tissues [10] and cells [6,11], and has recently received renewed attention as a tool for monitoring real-time metabolic activity at the level of multiple and the single eukaryotic cells [12]. IR microscopy allows the study of even complex biospecimens without the need of chemical modification such as molecular labelling, staining, or other sample alterations, thus making it a fully non-invasive untargeted technique [13]. It can be performed on both adhered [14] and suspended [15] cells, allowing the study of both biologically relevant states.

FTIR spectroscopy on macroscopic samples, while allowing the screening of extended samples with better signal-to-noise ratio and higher sensitivity than its microscopy implementation, also delivers ensemble averaged values. It is therefore unable to inform about the properties of individual cells, to relate cellular properties to spatial location and/or morphology, to inform on low abundance phenotypes, or to discriminate cellular from extracellular properties.

Here, we experimentally test the capability of IR microscopy to monitor individual cells from the same line but with widely different morphologies. We used a HEK-derived cell line [16] to assess whether it is possible to discriminate the metabolic turnover in cell types with widely different morphology, namely adherent and suspended cells. We benchmarked the applicability of IR microscopy using both synchrotron radiation and thermal sources and demonstrated that the method allows detecting the distinct metabolic activity of the two cell morphologies. This conclusion will be used in future work aiming to investigate the properties of specific cells and their environment within complex cellular assemblies, where the identity, morphology and cell cycle phase of individual cells can vary in space and time.

\section{Results and Discussion}

We performed a systematic study of HEK-cFXN single-cells in both adherent and suspended conditions. Cells were enclosed in the sample holder (described in Supporting information), isolated from the atmosphere, and monitored under the IR microscope over a 25-min interval without circulating fresh medium. The evolution of their biochemical and biophysical properties was assessed by recording spectral variations in the range of $1000 \mathrm{~cm}^{-1}-3050 \mathrm{~cm}^{-1}$ using both synchrotron radiation and a thermal source (Figure 1). The optic geometry implied that, in the case of the suspended cells, the footprint of the IR microbeam from the thermal source covered a whole single cell plus part of the surrounding medium. The suspended cells were about $10 \mu \mathrm{m}$ thick, i.e., comparable to the spacer thickness, and were sandwiched in contact with the two optical windows, leaving 
no headspace. Adherent cells were stretched over the surface, with an approximately polygonal shape, and estimated thickness between $2 \mu \mathrm{m}$ to $5 \mu \mathrm{m}$. Therefore, they were thinner than the $10 \mu \mathrm{m}$ spacer used in the sample holder, leaving a corresponding headspace which was also probed by the IR beam. In the case of adherent cells, most of the cell core was probed in the measurement, except for the longer cellular extensions. Figure 1 highlights the spectral regions analysed. The fingerprint region contains absorption bands from most of the molecular components of the cells. The fine variations in band intensity in this region, if decoded, can provide detailed information on all the ongoing chemical processes within the cell and its immediate environment [17]. The higher wavenumber region mostly displays absorption bands from $\mathrm{C}-\mathrm{H}$-containing molecules, plus carbon dioxide, within the sample and the atmosphere. The interval of the absorption band from the water bending mode, between $1680 \mathrm{~cm}^{-1}$ and $1610 \mathrm{~cm}^{-1}$, was removed in the displayed spectra because of the high noise levels.

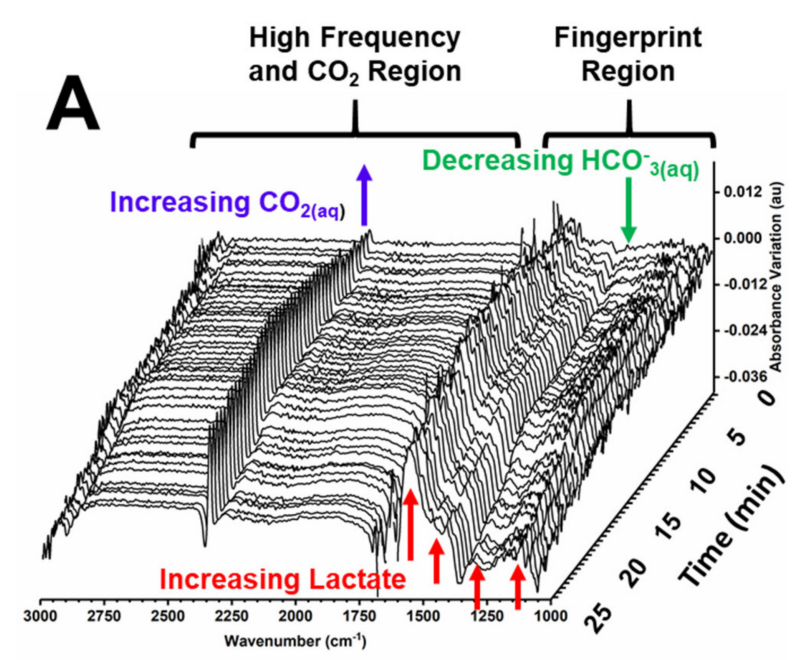

\section{Adherent Cell}

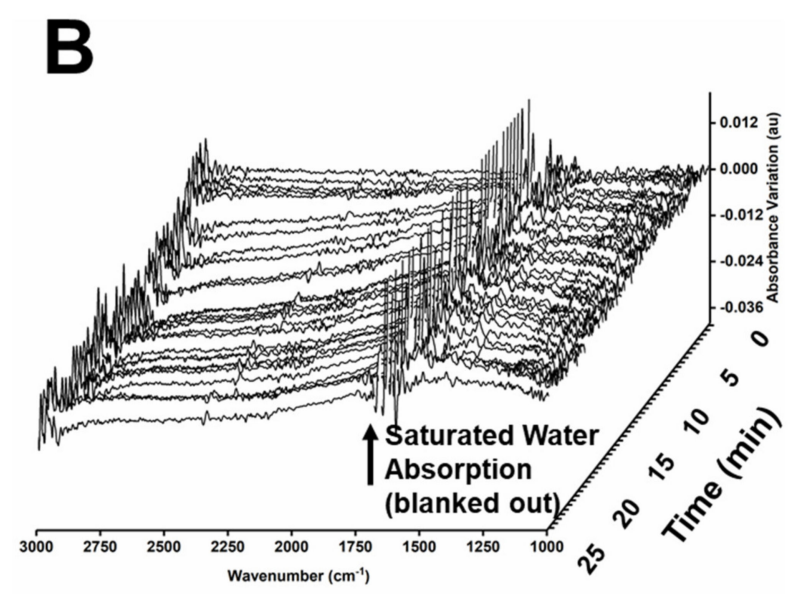

Suspended Cell

Figure 1. Spectral variations of single adherent and suspended cells over $25 \mathrm{~min}$. (A). Single Adherent Cell. The brackets define the fingerprint spectral region and the high frequency region. The arrows show the increasing absorption bands from lactate (red) and $\mathrm{CO}_{2(\mathrm{aq})}$ (blue) and the decreasing absorption band from $\mathrm{HCO}_{3}{ }^{-}$(aq) (green). (B). Single Suspended Cell. The arrow shows the position of the saturated absorption band from water, dominated by noise (points removed from both $\mathrm{A}$ and B for clarity). 
The spectral variations displayed by the adherent and suspended cells were remarkably different, with suspended cells showing small irregular variations over time, while clear changes were observed in the spectra of the adherent cells. When observed, the variations were dominated by the increase and decrease of multiple bands in the fingerprint region. By recording the cell position at the beginning and at the end of each measurement, we could associate many of the observed variations to cellular movements, and/or to changes in cell morphology, such as swelling, all of which can perturb IR spectra by changing the amount of material probed by the beam. For this reason, we restricted our analysis to the dominant spectral changes. In addition, we discarded cells that displayed major structural rearrangements or displacement over the course of the experiment to perform quantitative assessments.

The most obvious spectral difference between the two cell morphologies was the increase in absorption from aqueous carbon dioxide $\left(\mathrm{CO}_{2(\mathrm{aq})}\right)$, which dominated the spectra of adherent cells, while being absent from the spectra of suspended cells (Figure 1). The $v_{3}$ absorption band of $\mathrm{CO}_{2(\mathrm{aq})}$, observed at $2343 \mathrm{~cm}^{-1}$, overlapped with the interfering absorption from the atmospheric carbon dioxide $\left(\mathrm{CO}_{2(\mathrm{~atm})}\right)$, which had to be removed by subtraction. Observed variations in absorbance units, in the range of 2-3 mAU over $25 \mathrm{~min}$, can be converted to concentration changes using published absorption coefficients $\left(1.5 \times 10^{-4} \mu \mathrm{m}^{-1} \mathrm{mM}^{-1}\right)$ [18], indicating an increase of $\mathrm{CO}_{2(\mathrm{aq})}$ concentration in the $20 \mathrm{mM}$ range in the proximity of single adherent cells.

In parallel to the increase of $\mathrm{CO}_{2(\mathrm{aq})}$ absorption, a decrease in an absorption band at $1361 \mathrm{~cm}^{-1}$ was observed in adherent cells. The band was attributed to a diminution in the concentration of aqueous bicarbonate $\left(\mathrm{HCO}_{3}{ }^{-}{ }_{(\mathrm{aq})}\right)$. The $\mathrm{HCO}_{3}{ }^{-}{ }_{\text {(aq) }}$ absorption falls in the much more crowded fingerprint region than the absorption band of $\mathrm{CO}_{2(\mathrm{aq})}$. Nonetheless, variations were generally larger than any other surrounding changes, allowing an estimate of the absorption change. Furthermore, when observed, the variations were always negative and unaffected by cellular movements, most likely indicating that they correspond to a decrease of the extracellular bicarbonate concentration. Using an absorption coefficient of $0.4 \times 10^{-4} \mu \mathrm{m}^{-1} \mathrm{mM}^{-1}$ [18], we estimated variations in concentration in the $20 \mathrm{mM}$ range over $25 \mathrm{~min}$, comparable to the variation of $\mathrm{CO}_{2(\mathrm{aq})}$. (Figure 2) The correspondence and magnitude of the variations exclude major contributions from extracellular reactions or non-physiological processes, such as the dissolution of atmospheric $\mathrm{CO}_{2}$.

Concerted variations in the concentration of $\mathrm{HCO}_{3}{ }^{-}$(aq) and $\mathrm{CO}_{2(\mathrm{aq})}$ indicated that the formation of $\mathrm{CO}_{2}$ was caused by the protonation of bicarbonate, corresponding to a $\mathrm{pH}$ decrease. The protonation of bicarbonate forms carbonic acid, which rapidly dissociates into $\mathrm{CO}_{2(\mathrm{aq})}$ and water [18]. The volume probed by the infrared beam includes the cytoplasmic space and the extracellular space, implying that measured spectroscopic changes describe the average response over the two volumes. In the assumption that the buffering capacity is dominated by bicarbonate, the local $\mathrm{pH}$ value following acidification could be estimated from the change in concentration of $\mathrm{CO}_{2(\mathrm{aq})}$ and $\mathrm{HCO}_{3}{ }^{-}$(aq) [17], corresponding to final $\mathrm{pH}$ values of approximately 5.9 to 6.1 .

Extracellular acidification is the standard reporter of a cellular metabolism dominated by glycolysis and pyruvate fermentation. Fermentation of pyruvate generates lactate as the final product of the catabolism of glucose, which is then secreted into the extracellular environment. Lactate secretion is usually the direct cause of extracellular acidification via the action of the monocarboxylate transporter, which transfers one proton per lactate molecule to the extracellular environment. Accordingly, the measured acidification in these experiments was comparable to the values we had previously reported from IR microscopy of A549 cell cultures [17]. 

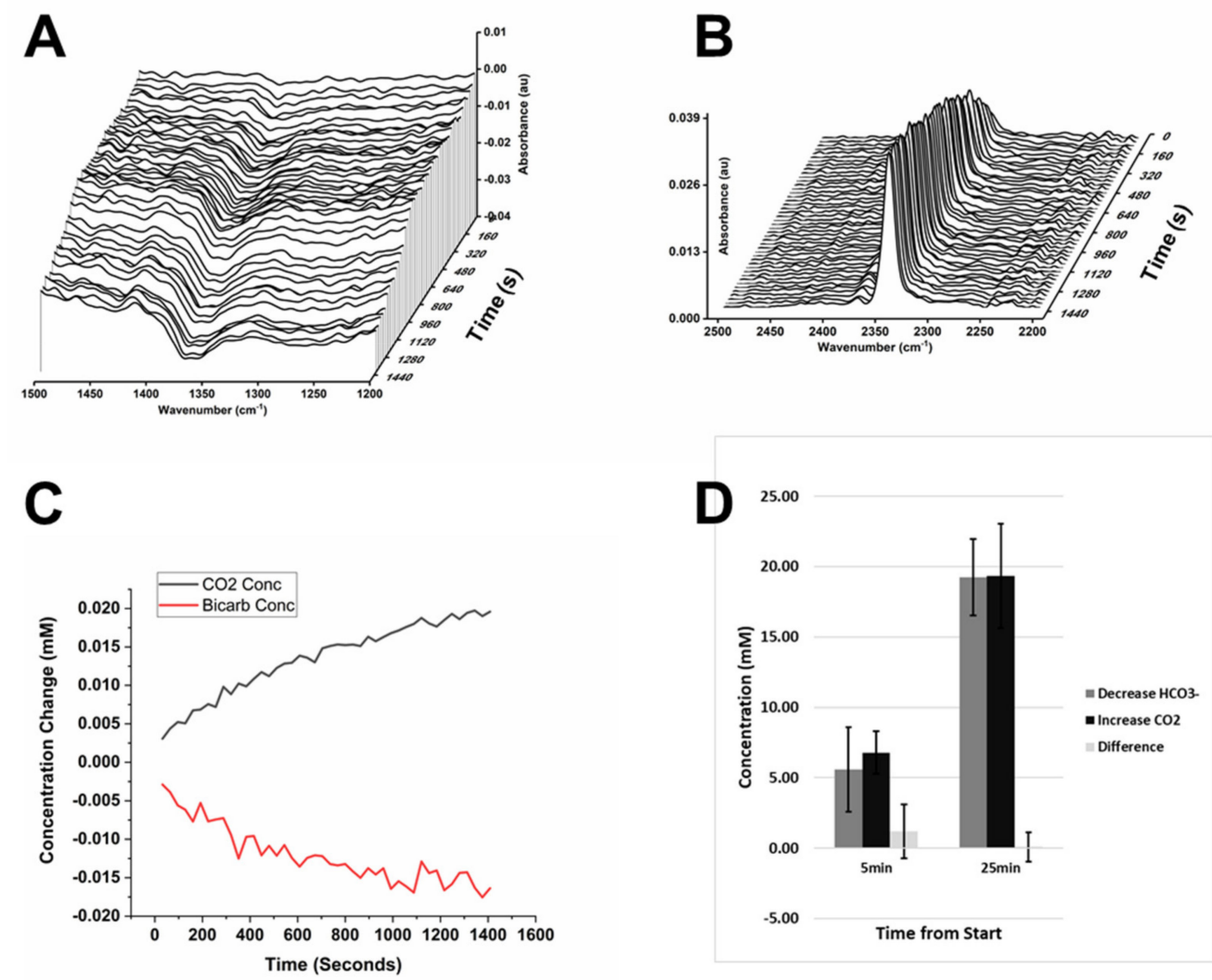

Figure 2. Comparison of formation of $\mathrm{CO}_{2(\mathrm{aq})}$ and consumption of $\mathrm{HCO}_{3}{ }^{-}$(aq) in single cell measurements. (A). Decrease of the $\mathrm{HCO}_{3}{ }^{-}$(aq) absorption band at $1361 \mathrm{~cm}^{-1}$. (B). Increase of the $\mathrm{CO}_{2(\mathrm{aq})}$ absorption band at $2343 \mathrm{~cm}^{-1}$. (C). Changes in the concentration of $\mathrm{CO}_{2(\mathrm{aq})}$ and of $\mathrm{HCO}_{3}{ }^{-}$(aq) corresponding to the absorption changes in $\mathrm{A}$ and $\mathrm{B}$. (D). Comparison of $\mathrm{CO}_{2(\mathrm{aq})}$ formation and $\mathrm{HCO}_{3}{ }_{(\mathrm{aq})}$ consumption in individual adherent HEK-cFXN cells with normal expression of frataxin and the difference between $\mathrm{CO}_{2(\mathrm{aq})}$ formation and $\mathrm{HCO}_{3}{ }^{-}$(aq) consumption calculated for each cell. Means from three cells (error bars show $\pm \sigma$ interval).

To confirm that acidification prevalently arose from glycolytic metabolism, we analyzed the spectra for the presence of lactate. The fingerprint region of adherent cells displayed a wealth of small absorption changes, arising from multiple cellular processes. The analysis of the pattern of these variations in the spectra of monolayers of A549 cells had previously revealed the formation and consumption of small molecules, including lactate [17]. A similarly detailed analysis was not feasible on the single cell samples analyzed in the present work because of the lower signal-to-noise ratio obtained in single cell spectra. Nonetheless, bands attributable to lactate became detectable in the spectra of single adherent cells after measuring for several minutes, although with low intensity. Comparison with the spectrum of sodium lactate, confirmed the identification of multiple lactate bands $\left(1573 \mathrm{~cm}^{-1}, 1413 \mathrm{~cm}^{-1}, 1314 \mathrm{~cm}^{-1}, 1125 \mathrm{~cm}^{-1}, 1043 \mathrm{~cm}^{-1}\right)$. The lactate bands increased over time, in parallel with the decrease of the band of bicarbonate $\left(1360 \mathrm{~cm}^{-1}\right)$ and the increase of the band of $\mathrm{CO}_{2(\mathrm{aq})}\left(2343 \mathrm{~cm}^{-1}\right)$ (Figure 1). To corroborate the identification, we improved the signal-to-noise ratio by averaging three spectra of single adherent cells (Figure 3). A positive variation of the bands was observed in multiple cells. The average local lactate variation over $25 \mathrm{~min}$ was estimated to be in the millimolar range by comparison with the spectra of sodium lactate. This was comparable, although somewhat lower, to the observed formation of $\mathrm{CO}_{2(\mathrm{aq})}$ and consumption of $\mathrm{HCO}_{3}{ }^{-}$(aq), thus reinforcing the correlation between acidification and lactic fermentation. 


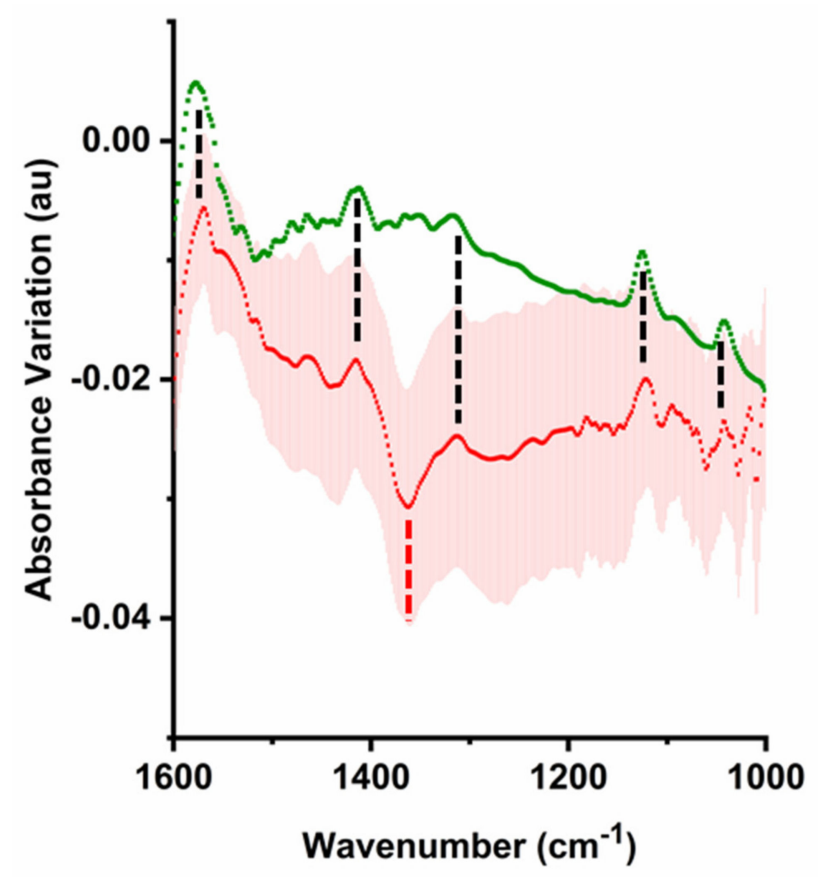

Figure 3. Detection of Lactate Formation. Comparison of the differential absorbance in the fingerprint region after $25 \mathrm{~min}$ averaged over 3 cells (the red line shows the mean of the spectra, while the shaded area shows values of $\pm \sigma$ at each wavenumber), with the absolute absorbance of $10 \mathrm{mM}$ sodium lactate (green line). The dashed black lines highlight increasing lactate absorption. The dashed red line highlights decreasing bicarbonate absorption.

In addition to being generated by acidification of the medium, production of detectable $\mathrm{CO}_{2(\mathrm{aq})}$ was expected from turnover within the citric acid cycle and from other metabolic pathways [19]. In our experiments, the increase of $\mathrm{CO}_{2(\mathrm{aq})}$ in adherent cells was closely matched to the consumption of $\mathrm{HCO}_{3}{ }^{-}{ }_{(\mathrm{aq})}$, indicating a negligible contribution from metabolic $\mathrm{CO}_{2(\mathrm{aq})}$ production. Surprisingly, no $\mathrm{CO}_{2(\mathrm{aq})}$ was detected in measurements on suspended cells, even during the first few minutes of the measurements.

The measurements reported in the previous sections were repeated using synchrotron radiation IR. The synchrotron infrared beam can be focused to a diffraction-limited light spot via the same microscope optics used for the thermal source. The smaller dimensions of the synchrotron IR spot allowed selective probing of suspended cells with minimal contribution from the surrounding medium. Measurements with the synchrotron source confirmed the difference in $\mathrm{CO}_{2(\mathrm{aq})}$ and $\mathrm{HCO}_{3}{ }^{-}$(aq) turnover already reported between adherent and suspended cells. However, they also revealed an unexpected optical effect not observed with the thermal source.

A distinguishing feature of all spectra recorded with synchrotron light, from both adherent and suspended cells, was the contribution from baseline variations that evolved over time (Figure 4). The variations appeared as broad spectral fluctuations, with a variable apparent period of the order of several hundred wavenumbers (ca. $700-1000 \mathrm{~cm}^{-1}$ ), and extending throughout the mid-IR spectral region, including the $1800-2500 \mathrm{~cm}^{-1}$ interval where biological matter typically shows few or no IR-active modes. In all cases, they developed progressively during the measurement, as the microbeam was maintained in the same sample position. However, they were not seen if the beam position was translated during the experiment. They were exclusively linked to cell presence and were not observed when measuring the medium in the sample holder, thus suggesting an interplay between the properties of the cellular matter and specific characteristics of the IR source. We did not observe oscillations with such structure when measuring dried cells. The latter observation, together with the observed time evolution, indicated that they arise from the properties of intact cells following protracted exposure to the synchrotron beam. 


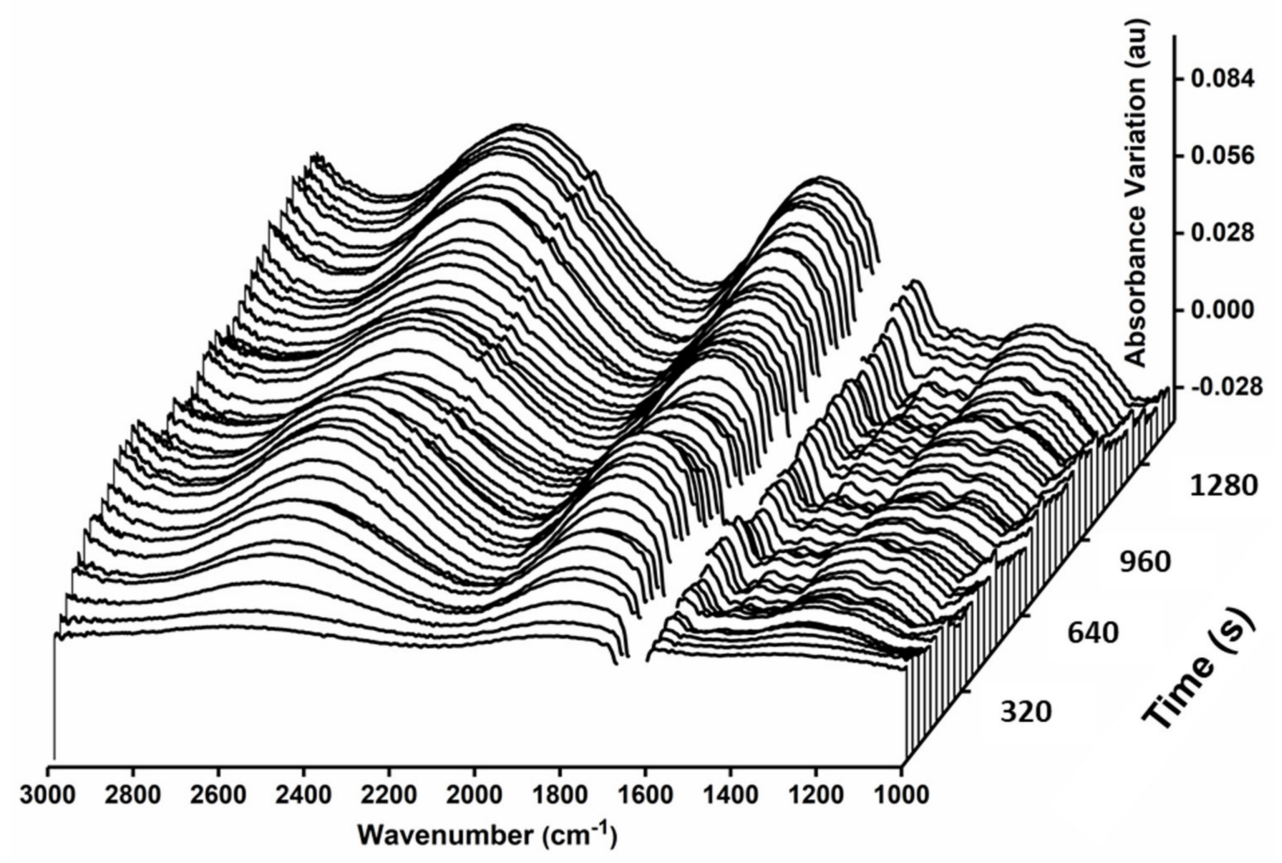

Figure 4. Effect of Synchrotron radiation on Single Cells. Time course of the spectrum of a single suspended cell when measuring with synchrotron radiation. Spectra measured at $32 \mathrm{~s}$ intervals over a total of $25 \mathrm{~min}$. The interval between $1600 \mathrm{~cm}^{-1}$ and $1700 \mathrm{~cm}^{-1}$ shows saturation of water absorption and has been removed for clarity.

When compared to radiation from a thermal source, synchrotron radiation is mainly characterized by a uniquely broadband spectrum, including the whole IR region, a greater degree of spatial and temporal coherence, and a pulsed time structure. Diamond beamline B22, used in these experiments, extracts radiation over the 10,000 to $5 \mathrm{~cm}^{-1}$ spectral range, which is limited by the FTIR beam splitter and filter used in our specific experiment to allow only infrared radiation to reach the sample. The fluctuations could be interpreted as interference fringes from variations in the real part of the refractive index of the cell during the measurement, perhaps enhanced by the coherence of the synchrotron emission [20]. Our first hypothesis is that such spectral oscillations could be related to dynamic changes of the refractive index and geometrical properties of the multiple layers of material crossed by the microbeam. Optical variations involving the headspace between cell and optical windows (for adherent cells) and the properties of subcellular structures, including the nucleus, membrane stacks, coacervates and organelles, could all contribute to these effects. The observation of the optical effect with both adherent and suspended cells, which have widely different geometry, with no headspace present for the latter, proved that it is related to cellular presence under the beam, and not to the geometry of the headspace.

An alternative, or additional, interpretation is that the observed fluctuations could arise from changes in collective vibrations within the cell, such as the absorption of hydrogen bonded networks of water molecules [21,22], and/or from coupling to vibrations of the cellular skeleton or of the membranes. Both interpretations require the cells to be intact, highlighting the potential usefulness of these oscillations, which become visible in IR microscopy by the use of a diffraction limited, pulsed, and coherent source, as a physical marker of cellular viability.

Finally, we validated the results by assessing possible differences in glycolysis, the glycolytic capacity, and the glycolytic reserve between adherent and suspension HEKcFXN cells by measuring the extracellular acidification rate (ECAR) by a Seahorse XF96 extracellular flux analyser (Agilent). The experiment, termed glycolytic stress assay, relies on monitoring the variation of extracellular $\mathrm{pH}$ during the sequential addition of glucose, oligomycin (ATP synthase inhibitor) and 2-deoxy-glucose (2-DG, a glucose analogue) compounds to the medium of glucose-starved cells. Glycolysis under basal conditions and 
glycolytic capacity of suspension cells compared to adherent cells were unaffected while we detected a strong, statistically significant $(p \leq 0.01) 73 \%$ reduction in glycolytic reserve of suspended as compared to adherent cells (Figure 5). The glycolytic reserve, calculated as the difference in ECAR between the glycolytic capacity and basal glycolysis, is a measure of the cell's ability to compensate through glycolysis when there is an increase in energy demand. Our results indicated a reduced ability of suspended cells to compensate for high energy demand compared to adherent cells, independently confirming the FTIR results.
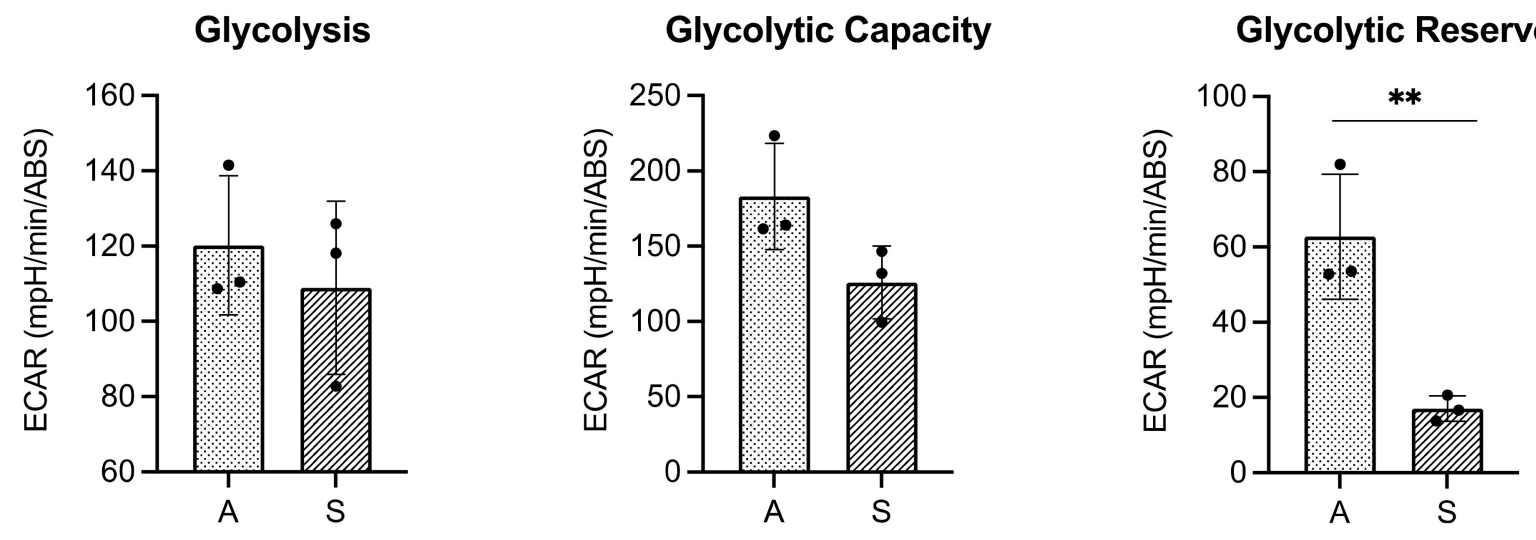

Figure 5. Glycolytic stress assay to validate the FTIR results. The assay was performed by measuring ECAR variations of adherent (A) and suspended (S) cells during time. Glycolysis, glycolytic capacity, and glycolytic reserve were obtained by sequentially treating the samples with glucose and oligomycin. Data are means \pm SD of $n=3$ independent experiments. Each experiment is expressed as the mean of three technical replicates, each replicate being composed by 12 biological samples $\left(1 \times 10^{5}\right.$ cells per sample). The recorded signals were normalized against total protein content (expressed as an absorbance value, ABS). A positive variation corresponds to a decrease of $\mathrm{pH}$. Data were analysed by t-test $\left.{ }^{* *} p \leq 0.01\right)$.

\section{Conclusions}

In this work, we used IR microscopy to monitor the metabolic activity of suspended versus adhered living HEK-derived cells by relying on the intrinsic IR light absorption from multiple molecular species in the sample. By monitoring variations in the concentration of $\mathrm{CO}_{2}, \mathrm{HCO}_{3}{ }^{-}$, and lactate, we quantified the metabolic activity at the single cell level, revealing large metabolic differences between adherent and suspended cells. Whereas adherent cells displayed the hallmarks of a metabolism relying on glycolysis followed by lactic fermentation, suspended cells did not display any metabolic activity within the detection limits of IR microscopy. We also identified a spectral effect that appears to arise from the interplay of synchrotron infrared radiation and the cells and manifests itself as oscillations in the spectral domain that slowly develop over time. The effect deserves further investigation to assess its potential application as a marker for intact or functional cells and could be developed into a tool for monitoring the cellular state.

Following the track of the present work, IR microscopy could be translated into assessing the relationship between adhesion state and metabolism in the broader biological and biomedical research contexts. Within this perspective, our methodological work intends to set a reliable background for IR microscopy studies of cellular metabolism. By addressing its application to different morphological states of individual cells, we open the way to the characterization of more complex heterogenous cellular assemblies, with insight that would be inaccessible via bulk measurements.

\section{Materials and Methods}

\subsection{HEK-cFXN Cell Line}

The HEK-cFXN cell line used in these experiments has been described previously [16]. Briefly, the cell line was derived for other purposes from the immortalized commercial HEK line via knockout of the endogenous FXN gene, which expresses the protein frataxin, and replacing it with an exogenous, inducible, FXN gene to allow the fine-tuning of the frataxin 
levels. For the present work, the capability of this cell line to modulate frataxin expression was not exploited, and the conditions were tailored to result in physiological levels of the protein. We used these cells only as a convenient and already well-characterized model system.

HEK-cFXN cells were cultured in Dulbecco Modified Eagle Medium (DMEM), supplemented with $10 \%$ tetracycline-free foetal bovine serum (FBS; Clontech, Takara, Mountain View, CA), $10 \mathrm{mM}$ sodium pyruvate, $2 \mathrm{mM}$ L-glutamine, $2 \%(v / v)$ non-essential amino acids (NEAAs), $100 \mu \mathrm{g} / \mathrm{mL}$ hygromycin B, and $15 \mu \mathrm{g} / \mathrm{mL}$ blasticidin. Cells were cultured in an incubator at $37{ }^{\circ} \mathrm{C}$ with humidified air and $5 \% \mathrm{CO}_{2}$. Unless differently stated, all reagents were acquired from ThermoFisher Scientific (Waltham, MA, USA). Sodium lactate was purchased from SIGMA-Aldrich (Sigma-Aldrich Chemie GmbH, Buchs, Switzerland).

\subsection{Sample Preparation}

HEK-cFXN cell samples were cultured in an incubator with a controlled atmosphere at $5 \% \mathrm{CO}_{2}$ and only transferred into the sample holder for IR microscopy immediately before the measurement. FTIR spectra were acquired using either suspended or adherent HEKcFXN cells. For suspended cell samples, HEK-cFXN cells were cultured overnight in 6-well plates and then prepared, immediately before the experiment, by detaching them with trypsin (Trypsin-EDTA $0.05 \%$, Thermo Fisher Scientific, Waltham, MA) treatment for circa $30 \mathrm{~s}$. Detached cells were resuspended in DMEM medium. The solution $(10 \mu \mathrm{L})$ containing cells was transferred into the sample holder, namely, a liquid cell for IR microscopy (see Supplementary Information). For adherent cell samples, HEK-cFXN cells were cultured overnight on $\mathrm{CaF}_{2}$ windows, allowing them to adhere to the window surface. The day after, the $\mathrm{CaF}_{2}$ window with adhered cells was assembled with the other components of the sample holder. The empty volume created by the spacer between the two $\mathrm{CaF}_{2}$ windows (Crystran, Poole, UK) was filled with $10 \mu \mathrm{L}$ DMEM medium. All samples were analysed by IR microscopy acquisition immediately after preparation in the sample holder. Once the cells were placed in it, no fresh medium was introduced, and the cells relied on the nutrients present in the volume of medium enclosed between the windows. The cell was sealed to prevent exchange of gas with the atmosphere. For the duration of the experiment, the sample holder was kept at $37^{\circ} \mathrm{C}$.

\subsection{IR Microscopy Instrumentation}

IR microscopy was performed at the MIRIAM beamline B22 of (Diamond Light Source, Didcot, UK) on a Hyperion 3000 IR microscope coupled to a Vertex $80 \mathrm{~V}$ interferometer (Bruker, Ettlingen, Germany), using a high sensitivity single element MCT (Mercury Cadmium Telluride) detector with size 50 microns and spectral cut off at ca. $650 \mathrm{~cm}^{-1}$. A broadband $\mathrm{KBr}$ beam splitter was used in the FTIR interferometer for covering the near/mid-IR spectral region (approx. 8000 to $400 \mathrm{~cm}^{-1}$ ). A Ge multilayer-coated filter was used in the optical path of the microscope to remove higher energy photons, thus defining the microscope illumination and spectral detection only in the mid-IR range (below circa $4000 \mathrm{~cm}^{-1}$ ). The IR beam was focused using a Schwarzschild condenser with $36 \times$ magnification and a numerical aperture 0.5 . All measurements were performed in transmission mode, using a $36 \times$ objective identical to the condenser. The microscope was used in apertureless mode for all measurement by either synchrotron IR from beamline B22 or the thermal source (black body at circa $1000 \mathrm{~K}$ ) inside the FTIR. The diameter and depth of focus of the diffraction-limited synchrotron light spot are a function of the IR wavelength [23]. At $6 \mu \mathrm{m}$, in the middle of the spectral range used in these experiments, the lateral resolution provided by a NA $=0.5$ objective is circa $6 \mu \mathrm{m}$, smaller than the lateral dimension of both adherent and suspended cells. The corresponding depth of focus is circa $35 \mu \mathrm{m}$, a few times greater than the thickness of the $10 \mu \mathrm{m}$ aqueous layer, implying that the expected lateral distribution of the beam is practically uniform across the sample. The light spot of the thermal source was not diffraction-limited under the conditions of operation, neither transversally nor longitudinally, and it is expected to be 
larger than the size of both adherent and suspended cells for the microscope optics used in these experiments. In agreement with these predictions, the integrated intensity of the microbeam over the full transmitted spectral range gave a Full-Width at Half Maximum (FWHM) of the detected spot of circa $15 \mu \mathrm{m}$ at the sample plane (data not shown) by the diffraction limited synchrotron radiation source, and approximately $25 \mu \mathrm{m}$ by the thermal source. Measurements were performed using either the synchrotron or the thermal source, depending on the required spatial distribution of the focal spot.

A custom sample holder for IR microscopy in aqueous environment (Suppl. Information and Suppl. Figure S1) was positioned on the automated motorized stage of the IR microscope to allow for sample inspection and measurement. The cells were enclosed in the holder and the visible light illumination of the microscope was used to inspect the sample with the help of a visible camera. Observation under visible light was used to assess sample movement.

\subsection{Spectral Measurements and Analysis}

We analyzed the data in terms of spectral variations, plotting the differential absorbance, rather than using the absolute spectra, because they highlight only the spectroscopic components changing over time (Note: Similar investigations of time-resolved changes by IR absorption spectroscopy reported in the literature often use the expression difference spectra, instead of spectral changes. The protocol used in these investigations often involves recording a background trace, followed by recording a sample trace in the sample location to obtain an absolute absorbance spectrum. A series of time-resolved absolute spectra is then recorded in the sample position, and the difference between the spectrum at time $t$ and the spectrum at time 0 is used to calculate the difference spectrum at time $t$. This is analytically equivalent to the results of the measurement as performed in the present work, so that the terms difference spectra and spectral changes are equivalent). Individual cells were selected through their visible morphology as observed by the microscope visible light. In the case of adherent cells, we targeted the ones that appeared fully adherent to the surface with multiple filopodia and focused the beam on the central part of the cell. An initial spectral measurement was performed on the cell at time zero $(t=0)$, followed by repeated consecutive spectral measurements (single channel sample). The IR microbeam was maintained at the same sample location throughout the measurement for each cell. The single measurements were used to calculate spectral changes over time on the absorbance scale (see note). In contrast, absolute absorbance spectra were recorded by measuring the background spectrum on the medium and the sample spectrum on the cell itself.

FTIR scans were run in double-sided forward-backward acquisition mode at $4 \mathrm{~cm}^{-1}$ resolution using a nominal scan rate of $80 \mathrm{kHz}$ (referred to the HeNe laser frequency of ca. $\left.15,800 \mathrm{~cm}^{-1}\right)$. A total of 256 scans were recorded and summed up for the background and for each measurement, corresponding to a total collection time for each spectrum of $32 \mathrm{~s}$. Each cell was monitored for about 25 min after enclosure in the holder. The duration of the measurement was chosen to obtain an acceptable $\mathrm{S} / \mathrm{N}$ ratio for lactate bands. Single spectra were obtained by Fourier transforming the interferogram after apodization with a Blackman-Harris 3-term function, using a zero-filling factor of 4 and a Mertz phase correction. Data were plotted as absorbance variations from the initial state over the time of the measurement. Spectra of single cells were recorded over time and analysed for the mid-IR spectral region. The thickness of the medium layer, $10 \mu \mathrm{m}$, led to high noise levels around $1640 \mathrm{~cm}^{-1}$ due to the water strong IR absorption (water bending mode). As the thickness of the $\mathrm{CaF}_{2}$ windows (twice $1 \mathrm{~mm}$ ) reduced the usable spectral range to above $1000 \mathrm{~cm}^{-1}$, the IR spectral region appeared limited by noise at the extremes of the spectral interval, as well as in proximity of $1640 \mathrm{~cm}^{-1}$. Atmospheric changes (i.e., water vapour and gaseous carbon dioxide $\mathrm{CO}_{2(\mathrm{~g})}$ ) were removed by the automated atmospheric correction algorithm in OPUS, which left minimal residual variations of atmospheric $\mathrm{CO}_{2}$ bands. 
Quantitative measurement of $\mathrm{CO}_{2(\mathrm{aq})}$ concentrations in liquid was instead performed after manual subtraction of $\mathrm{CO}_{2(\mathrm{~g})}$ bands in gas phase.

Variations in the concentration of $\mathrm{CO}_{2(\mathrm{aq})}$ and $\mathrm{HCO}_{3}{ }^{-}$were determined using the absorption coefficients by Falk and Miller $\left(\varepsilon_{\left(2343 \mathrm{~cm}^{-1}\right)}=1.5 \times 10^{6} \mathrm{~cm}^{2} \mathrm{~mol}^{-1}\right.$ or $1.5 \times 10^{-4} \mathrm{\mu m}^{-1} \mathrm{mM}^{-1}$ for $\mathrm{CO}_{2}(\mathrm{aq})$ and $\varepsilon_{\left(1360 \mathrm{~cm}^{-1}\right)}=0.4 \times 10^{6} \mathrm{~cm}^{2} \mathrm{~mol}^{-1}$ or $\left.4 \times 10^{-5} \mu \mathrm{m}^{-1} \mathrm{mM}^{-1}\right)$ [19].

\subsection{Acidification}

An estimate of final $\mathrm{pH}$ in the $\left[\mathrm{CO}_{2(\mathrm{aq})}\right]$ and $\left[\mathrm{HCO}_{3}{ }^{-}\right]$buffer is provided from measured concentrations of using Equation (1):

$$
\mathrm{pH}=\mathrm{pK}_{\mathrm{a}}-\log \left(\left[\mathrm{CO}_{2(\mathrm{aq})}\right] /\left[\mathrm{HCO}_{3}{ }^{-}\right]\right)
$$

where $K_{a}$ is the apparent acid dissociation constant for the serial equilibria involving hydration of aqueous $\mathrm{CO}_{2(\mathrm{aq})}$ and dissociation of carbonic acid to bicarbonate. In this work, we use $\mathrm{pK}_{\mathrm{a}}=6.04$. $\left[\mathrm{CO}_{2(\mathrm{aq})}\right]$ was determined from the measured increase in $\mathrm{CO}_{2(\mathrm{aq})}$ in the IR beam. $\left[\mathrm{HCO}_{3}{ }^{-}\right.$] was estimated using an initial concentration of bicarbonate of $44 \mathrm{mM}$, as in the medium, and subtracting the amount of $\mathrm{HCO}_{3}{ }^{-}$(aq) consumed in the IR beam. $\left[\mathrm{CO}_{2(\mathrm{aq})}\right]$ was determined from the measured increase in $\mathrm{CO}_{2(\mathrm{aq})}$ in the IR beam under the assumption that the initial concentration of $\mathrm{CO}_{2(\mathrm{aq})}$ in the medium was negligible. Equation (1) is valid in the $\mathrm{pH}$ range where buffering capacity is dominated by $\mathrm{CO}_{2(\mathrm{aq})}$ and $\mathrm{HCO}_{3}{ }^{-}{ }_{(\mathrm{aq})}$, providing reliable values in the proximity of $\mathrm{pH}$. Outside this range, multiple equilibria between other species should be considered. The equation also assumes that the local buffering equilibrium is established much faster than diffusion processes away from the probed volume [24].

\subsection{Seahorse Experiments}

One day ( $24 \mathrm{~h}$ ) before the experiment, HEK-cFXN cells (adherent cell samples) were plated into Seahorse 96-well XF cell culture microplates (cat. no. 101085-004) at a density of $5 \times 10^{4}$ cells per well. Cells were left overnight at $37{ }^{\circ} \mathrm{C}$ in humidified air supplemented with $5 \% \mathrm{CO}_{2}$. The next day, the medium was changed to XF DMEM Medium (cat no. 103575-100) supplemented with $2 \mathrm{mM}$ Glutamax (the $\mathrm{pH}$ of the medium was adjusted to 7.4 using $1 \mathrm{M} \mathrm{NaOH}$ ). Cells were then incubated for $1 \mathrm{~h}$ at $37^{\circ} \mathrm{C}$ in a $\mathrm{CO}_{2}$-free incubator. Suspension cells were prepared on the day of the experiment by incubating adherent HEK-cFXN cells in XF DMEM Medium (supplemented with Glutamax) for 45 min, detached with trypsin and, immediately before the measurement, deposited in the 96-well XF cell culture microplate at a density of $1 \times 10^{5}$ cells per well. The 96-well XF cell culture microplate was pre-treated with Cell-Tak $(22.4 \mu \mathrm{g} / \mathrm{mL}$, Corning cat. no. 354240) to facilitate immobilisation of both suspension and adherent cells to the bottom of the microplate wells. The XF Glycolytic Stress Test (cat no. 103020-100) was carried out by loading the XF 96 Extracellular Flux assay sensor cartridge with glucose (final well concentration $10 \mathrm{mM}$ ), oligomycin (final well concentration $1.5 \mu \mathrm{M}$ ), and 2-DG (final well concentration $50 \mathrm{mM}$ ). Readings were normalized against the total protein content of each biological sample using a standard BCA assay (Thermo Scientific, cat. no. 23227). A positive variation corresponds to a decrease of $\mathrm{pH}$. Statistical significance was tested using the unpaired t-test $\left.{ }^{* *} p<0.01\right)$.

\subsection{Data Reliability and Statistics}

The experiments described in the present manuscript are the resultant of more than two months of experimental time distributed over six years during which we explored several different aspects and changed the parameters to test different conditions. In the present manuscript we describe only the results relevant to the methodological aspects of the experiment, i.e. the possibility to report differences in the turnover of suspended and adherent cells and the effect of synchrotron radiation on cells with one level of FXN expression. Data collected at different levels of FXN expression back up and increase the statistics of our results, even though not directly discussed in the manuscript. 
Supplementary Materials: The following are available online at https://www.mdpi.com/article/10 .3390/ijms221910742/s1.

Author Contributions: T.V. took care of cell culture and sample preparation supported by G.M., L.Q., T.V. and A.d.R. performed the IR measurements, L.Q. and A.d.R. analysed the IR data. L.Q. prepared the graphical representation of IR data and of the IR setup and interpreted the IR results. T.V. performed Seahorse metabolic measurements, performed statistical analysis of Seahorse data, prepared their graphical representation and wrote their interpretation. G.C. managed and prepared the instrumentation, provided technical support and contributed to data interpretation. M.W. provided technical support and contributed to data interpretation. A.P. supervised the research, provided funding and contributed to interpretation and writing, L.Q. wrote the manuscript which was then revised by all the authors. All authors have read and agreed to the published version of the manuscript.

Funding: L.Q. was supported by the European Union's Horizon 2020 programme under the Marie Skłodowska-Curie grant no. 665778, managed by a POLONEZ project from the National Science Centre Poland (contract 2016/21/P/ST4/01321) and by an OPUS16 grant from the National Science Centre Poland (contract 2018/31/B/NZ1/01345). The research was supported by BBSRC and the UK Dementia Research Institute (RE1 3556) that is funded by Medical Research Council, Alzheimer's Society and Alzheimer's Research UK. The experiments were performed on beamline B22 of Diamond Light Source, UK, supported under the long-term proposal SM-18811. Preliminary experiments were performed at IFJ-PAN, Kraków, using equipment purchased under the Małopolska Regional Operational Program Measure 5.1. Project No. 2014/15/B/ST4/04839.

Institutional Review Board Statement: Not applicable.

Informed Consent Statement: Not applicable.

Data Availability Statement: Data are available from the authors upon reasonable request.

Acknowledgments: L.Q. is grateful to the Paul Scherrer Institut, Switzerland, for the construction and gift of the sample holder used in the present work. We are also grateful to the Institute of Nuclear Physics of the Polish Academy of Sciences for allowing instrumentation access for preliminary measurements.

Conflicts of Interest: The authors declare no conflict of interest.

\section{References}

1. Youssef, K.K.; Nieto, M.A. Glucose metabolism takes center stage in epithelial-mesenchymal plasticity. Dev. Cell 2020, 53, 133-135. [CrossRef]

2. Wang, Y.; Dong, C.; Zhou, B.P. Metabolic reprogram associated with epithelial-mesenchymal transition in tumor progression and metastasis. Genes Dis. 2020, 7, 172-184. [CrossRef]

3. Labuschagne, C.F.; Cheung, E.C.; Blagih, J.; Domart, M.C.; Vousden, K.H. Cell Clustering Promotes a Metabolic Switch that Supports Metastatic Colonization. Cell Metab. 2019, 30, 720-734.e5. [CrossRef]

4. Sato, M.; Kawana, K.; Adachi, K.; Fujimoto, A.; Yoshida, M.; Nakamura, H.; Nishida, H.; Inoue, T.; Taguchi, A.; Takahashi, J.; et al. Spheroid cancer stem cells display reprogrammed metabolism and obtain energy by actively running the tricarboxylic acid (TCA) cycle. Oncotarget 2016, 7, 33297-33305. [CrossRef] [PubMed]

5. Yang, Y.; Huang, Y.; Wu, J.; Liu, N.; Deng, J.; Luan, T. Single-cell analysis by ambient mass spectrometry. TrAC Trends Anal. Chem. 2017, 90, 14-26. [CrossRef]

6. Quaroni, L.; Zlateva, T. Infrared spectromicroscopy of biochemistry in functional single cells. Analyst 2011, 136, 3219-3232. [CrossRef] [PubMed]

7. Barer, R.; Cole, A.R.H.; Thompson, H.W. Infra-Red Spectroscopy with the Reflecting Microscope in Physics, Chemistry and Biology. Nature 1949, 163, 198-201. [CrossRef]

8. Cournoyer, R.; Shearer, J.C.; Anderson, D.H. Fourier Transform Infrared Analysis below the One-Nanogram Level. Anal. Chem. 1977, 49, 2275-2277. [CrossRef]

9. Levenson, E.; Lerch, P.; Martin, M.C. Infrared imaging: Synchrotrons vs. arrays, resolution vs. speed. Infrared Phys. Technol. 2006, 49, 45-52. [CrossRef]

10. Chiriboga, L.; Xie, P.; Yee, H.; Vigorita, V.; Zarou, D.; Zakim, D.; Diem, M. Infrared spectroscopy of human tissue. I. Differentiation and maturation of epithelial cells in the human cervix. Biospectroscopy 1998, 4, 47-53. [CrossRef]

11. Lasch, P.; Boese, M.; Pacifico, A.; Diem, M. FT-IR spectroscopic investigations of single cells on the subcellular level. Vib. Spectrosc. 2002, 28, 147-157. [CrossRef] 
12. Quaroni, L.; Zlateva, T.; Wehbe, K.; Cinque, G. Infrared imaging of small molecules in living cells: From in vitro metabolic analysis to cytopathology. Faraday Discuss. 2016, 187, 259-271. [CrossRef]

13. Bellisola, G.; Sorio, C. Infrared spectroscopy and microscopy in cancer research and diagnosis. Am. J. Cancer Res. 2012, 2, 1-21.

14. Kreuzer, H.W.; Quaroni, L.; Podlesak, D.W.; Zlateva, T.; Bollinger, N.; McAllister, A.; Lott, M.J.; Hegg, E.L. Detection of Metabolic Fluxes of $\mathrm{O}$ and $\mathrm{H}$ Atoms into Intracellular Water in Mammalian Cells. PLoS ONE 2012, 7, e39685. [CrossRef] [PubMed]

15. Goff, K.L.; Quaroni, L.; Wilson, K.E. Measurement of metabolite formation in single living cells of Chlamydomonas reinhardtii using synchrotron Fourier-Transform Infrared spectromicroscopy. Analyst 2009, 134, 2216-2219. [CrossRef] [PubMed]

16. Vannocci, T.; Faggianelli, N.; Zaccagnino, S.; della Rosa, I.; Adinolfi, S.; Pastore, A. A new cellular model to follow Friedreich's ataxia development in a time-resolved way. Dis. Model. Mech. 2015, 8, 711-719. [CrossRef] [PubMed]

17. Quaroni, L.; Zlateva, T. Real-time metabolic analysis of living cancer cells with correlated cellular spectro-microscopy. Anal. Chem. 2014, 86, 6887-6895. [CrossRef]

18. Falk, M.; Miller, A.G. Infrared spectrum of carbon dioxide in aqueous solution. Vib. Spectrosc. 1992, 4, 105-108. [CrossRef]

19. Naumann, D. FT-infrared and FT-Raman spectroscopy in biomedical research. Appl. Spectrosc. Rev. 2001, 36, 239-298. [CrossRef]

20. Chubar, O.; Elleaume, P.; Snigirev, A. Phase analysis and focusing of synchrotron radiation. Nucl. Instrum. Methods Phys. Res. Sect. A Accel. Spectrometers Detect. Assoc. Equip. 1999, 435, 495-508. [CrossRef]

21. Brzezinski, B.; Bartl, F.; Zundel, G. Excess proton hydrate structures with large proton polarizability, screened by tris(2-ethylhexyl) phosphate. J. Phys. Chem. B 1997, 101, 5607-5610. [CrossRef]

22. Leberle, K.; Kempf, I.; Zundel, G. An intramolecular hydrogen bond with large proton polarizability within the head group of phosphatidylserine. An infrared investigation. Biophys. J. 1989, 55, 637-648. [CrossRef]

23. Born, M.; Wolf, E. Principles of Optics: Electromagnetic Theory of Propagation, Interference and Diffraction of Light, 7th ed.; Cambridge University Press: Cambridge, UK, 1999; ISBN 0-521-64222-1.

24. Krieg, B.J.; Taghavi, S.M.; Amidon, G.L.; Amidon, G.E. In vivo predictive dissolution: Transport analysis of the $\mathrm{CO}_{2}$, bicarbonate in vivo buffer system. J. Pharm. Sci. 2014, 103, 3473-3490. [CrossRef] [PubMed] 